\title{
Observation of multiple chirping events in electron cyclotron emission of non-equilibrium mirror-confined plasma
}

\author{
M.E. Viktorov ${ }^{1,2}$, A.G. Shalashov ${ }^{1,2}$, D.A. Mansfeld ${ }^{1}$ and S.V. Golubev ${ }^{1}$ \\ ${ }^{1}$ Institute of Applied Physics Russian Academy of Sciences, Nizhny Novgorod, Russia, mikhail.viktorov@appl.sci-nnov.ru \\ ${ }^{2}$ Lobachevsky State University of Nizhny Novgorod, Nizhny Novgorod, Russia
}

Chirping frequency patterns have been observed in the electron cyclotron emission from a strongly nonequilibrium mirror-confined plasma created by powerful microwave radiation of gyrotron $(37.5 \mathrm{GHz}, 80 \mathrm{~kW})$ under electron cyclotron resonance (ECR) conditions [1]. An example of multiple chirping events in the spectrum of the electron cyclotron plasma emission is shown in fig. 1. Such patterns are typical for the formation of nonlinear phase-space structures in a proximity of the waveparticle resonances of a kinetically unstable plasma, also known as the "holes and clumps" mechanism $[2,3]$.

This mechanism has been extensively examined to study and interpret the Alfven wave turbulence driven by high-energy beam ions or alpha particles in toroidal magnetic traps which leads to the observation of the frequency sweeping events [4, 5]. The frequency sweeping events of energetic particle-driven Alfven instabilities were commonly observed on DIII-D, JT-60U, ASDEXUpgrade, MAST, NSTX, START, and LHD machines with NBI heating. Here, we report the first laboratory observations of potentially the same mechanism acting in a much higher frequency domain. The focus of this work is devoted to the study of peculiar time-frequency characteristics of the electromagnetic radiation, which has been made possible only recently with the advent of methods for measuring the electromagnetic field with high temporal resolution.

In the experiments, ambient plasma and a nonequilibrium component of energetic electrons are created under ECR conditions in the simple mirror trap. The hot electron fraction with a loss-cone distribution function is a matter of kinetic instabilities of various electromagnetic modes which are reviewed in [6]. External microwave heating operates for $1 \mathrm{~ms}$. Excitation of multiple chirping wave packets is observed after ECR heating switch-off with a delay from 0.1 to $1 \mathrm{~ms}$. The frequency band of the observed microwave emission is below the electron cyclotron frequency $f_{\text {ce } 0}$ in the trap center. The dependency of emission frequency band on the ambient magnetic field variations indicates the cyclotron nature of excited waves.

The microwave emission is observed only in a few narrow frequency bands that are independent of all experimental conditions. Isolated narrow frequency bands may be explained by excitation of individual modes of a bounded plasma in a discharge chamber [1]. When the magnetic field decreases such that $f_{\text {ce } 0}$ becomes lower than the corresponding frequency band, the microwave emission in this particular band is not detected anymore. Simultaneously, the microwave emission at lower frequency bands may still be detected or even triggered on in a new band.

Within each frequency band the dynamic spectrum of emission is a set of highly chirped radiation bursts with both increasing and decreasing frequencies, see fig. 1. Frequency variation rate is in the range $20-40 \mathrm{MHz} / \mu \mathrm{s}$. The bandwidth of this narrow-band emission is about $2 \times 10^{-3} f_{\text {ce } 0}$. Wave packets are grouped in batches with the number of bursts in packs of about 10 . The duration of narrow-band spike reaches about $10 \mu \mathrm{s}$. Bundles of these pulses occur quasi-periodically with a period of 10-20 $\mu$ s. Sometimes very long series of pulses with a duration up to $1 \mathrm{~ms}$ are observed.

The fine structure of the emission spectrum in a particular frequency band is changing with the ambient magnetic field decrease. This effect is clearly seen in fig. 2 for the case of a long series of chirping events. When $f_{\text {ce } 0}$ is significantly higher than emission frequency, we observe the predominance of rising tones with a sweeping frequency that saturates at more or less constant level for all neighboring pulses (fig. 2a). With the decrease of $f_{\mathrm{ce} 0}$, falling tones appear in the spectrum (fig. 2b). Finally, when $f_{\text {ce } 0}$ is close to the frequency of the microwave emission, a narrowband continuous signal without pronounced chirping patterns is observed (fig. 2c). Thus, the dynamic bandwidth of the emission is reduced with the decrease of difference between the electron cyclotron frequency and the emission frequency.

The "holes and clumps" mechanism, also known as Berk-Breizman model [2], seems to be the most suitable to explain the transients with periodic frequency sweeps observed in our experiment. As the mode grows, most of the particles respond adiabatically to the wave, and only a small group of resonant particles mix and cause local flattening of the distribution function in phase space within or near the separatrices formed by the waves. However, when linear dissipation from a background plasma is present, the saturated plateau state becomes unstable and the mode tends to grow explosively. That results in the formation and subsequent evolution of long-living, as compared to the linear growth, structures in the particle distribution, so-called holes (a depletion of particles) and clumps (an excess of particles). These structures represent nonlinear waves of Bernstein-Greene-Kruskal type whose frequencies are slightly up- and down-shifted with respect to that of the initial instability. Their subsequent convective motion in phase space is synchronized to the change in wave frequency, thus leading to complex chirping patterns in dynamical spectra of unstable waves [3]. A distinctive feature of the instability under investigation is the significant effect of electron collisions providing a dissipation channel for electromagnetic energy, which leads to the formation of characteristic chirped elements in the emission spectra. 

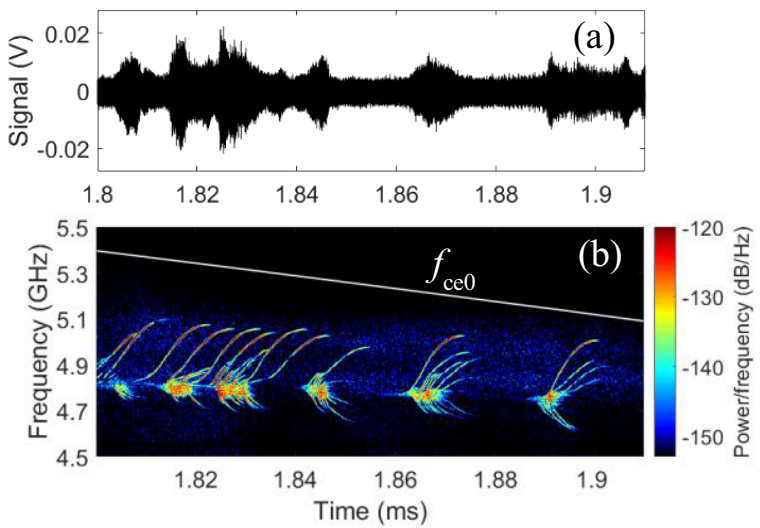

Fig. 1. (a) Waveform of the wave electric field oscillations and (b) its spectrogram. The white line shows the value of electron cyclotron frequency in the trap center on the axis. The ECR heating is operating during $0-1 \mathrm{~ms}$ (not shown). Adopted from [1]
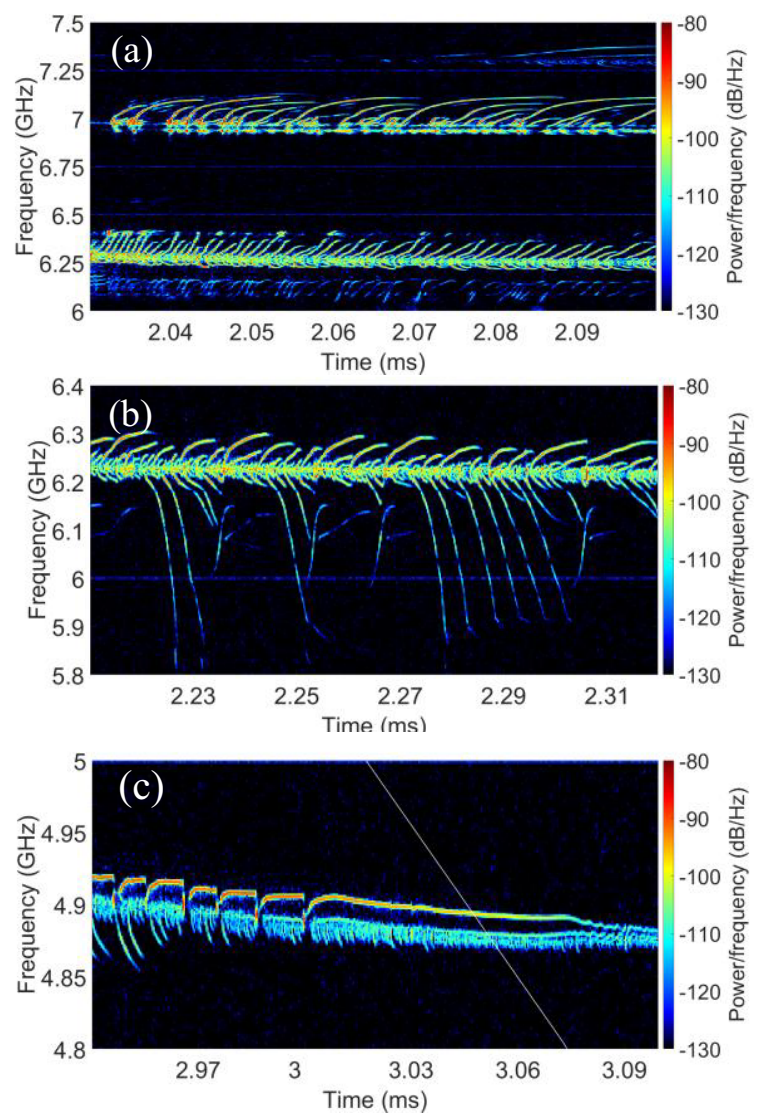

Fig. 2. Different regimes of chirping events in plasma emission spectrum observed during plasma decay. Panels $(a)-(c)$ correspond to different sequential time slots. The white line shows the value of electron cyclotron frequency in the trap center on the axis. The ECR heating is operating during $0-1 \mathrm{~ms}$ (not shown)

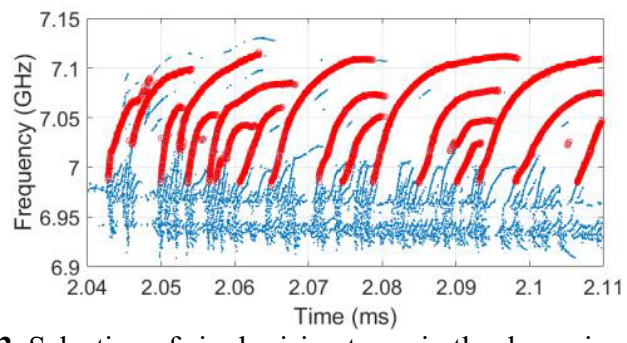

Fig. 3. Selection of single rising tones in the dynamic spectrum shown in fig. 2a (upper part)

For a very rough estimate, one can isolate the single spectral component (see fig. 3) and model the evolution in time of the chirping event. In the collisionless limit for resonant particles, a bounce average of Maxwell-Vlasov kinetic equations yields the frequency shift $\delta \omega$ of an unstable mode as [2]

$$
\delta \omega=\frac{16 \sqrt{2}}{3 \sqrt{3} \pi^{2}} \gamma_{L} \sqrt{\nu t} .
$$

This expression is valid for the regime where resonant particles are deeply trapped in the wave potential and perturbation of the passing particle is negligible. Fitting the measured frequency sweeps $\delta \omega=\sqrt{A t}$ by constant $A$ and assuming near-threshold condition $\gamma_{\mathrm{L}} \approx v$, one may estimate the upper boundary for the dissipation $v$ or, equivalently, the lower boundary for the growth rate $\gamma_{\mathrm{L}}$ compatible with (1). In the experiments we obtain typical values $A=(1-3) \times 10^{21} \mathrm{~s}^{-3}$ resulting in $\gamma_{\mathrm{L}}=(1.7-2.5) \times$ $\times 10^{7} \mathrm{~s}^{-1}$, what is in reasonable agreement with independent estimates. More details may be found in [1].

The work has been supported by RFBR (grants No. 16-02-00625, 16-32-60056).

\section{References}

1. Viktorov, M.E., Shalashov, A.G., Mansfeld, D.A., Golubev, S.V. Observation of quasi-periodic frequency sweeping in electron cyclotron emission of nonequilibrium mirror-confined plasma // EPL. 2016. V. 116. P. 55001.

2. Berk, H.L., Breizman, B.N., Petviashvili, N.V. Spontaneous hole-clump pair creation in weakly unstable plasmas // Phys. Lett. A. 1997. V. 234. P. 213.

3. Lilley, M.K., Breizman, B.N., Sharapov, S.E. Effect of dynamical friction on nonlinear energetic particle modes // Phys. Plasmas. 2010. V. 17. P. 092305.

4. Breizman, B.N., Sharapov, S.E. Major minority: energetic particles in fusion plasmas // Plasma Phys. Control. Fusion. 2011. V. 53. P. 054001.

5. Sharapov, S.E., et al. Energetic particle instabilities in fusion plasmas // Nucl. Fusion. 2013. V. 53. P. 104022.

6. Shalashov, A.G., Viktorov, M.E., Mansfeld, D.A., Golubev, S.V. Kinetic instabilities in a mirror-confined plasma sustained by high-power microwave radiation // Phys. Plasmas. 2017. V. 24. P. 032111. 